\title{
Technical Note: Tracking the Formation of Eumelanin from L-Dopa using Coupled Measurements
}

\author{
Philip Yip ${ }^{1,2} \&$ Jens U Sutter ${ }^{2 *}$ \\ 1 Horiba IBH Ltd. 133 Finnieston Street, Glasgow G3 8HB, Scotland, UK \\ 2 Photophysics Group, Department of Physics, John Anderson Building, \\ University of Strathclyde, Glasgow, G4 ONG, Scotland, UK \\ * jens.sutter@strath.ac.uk
}

\begin{abstract}
:
Melanin plays a crucial role as a pigment all through the animal kingdom. Being a macromolecule just on the divide between an ordered crystalline or a purely amorphous form melanin has proven a challenge to structure-function analysis. Melanin assembles from small molecules much like a jigsaw and much like in a jigsaw the fine detail quickly vanishes in the overall picture. With Melanin being first and foremost a photo-active molecule we focus on spectral properties for the characterization of its structure. We use absorption measurements to illustrate the complex nature of the formation process. To gain a better hold on the formation pathway we use coupled measurements of excitation and emission to identify 'areas of interest' in the Excitation-Emission Matrix (EEM). We then probe one area for characteristic fluorescence lifetimes to track one melanin building block through the formation process. Comparison of the EEMs of L-Dopa derived melanin with natural Sepia melanin shows characteristic differences. We show how the presence of copper ions creates a melanin closer to its natural form.
\end{abstract}

Introduction:

The formation of complex macromolecules from small precursor molecules is occurring in a range of diverse fields. Such processes are highly relevant to the oil- and gas-industry in the self-assembly of the raw materials ${ }^{1}$ or the polymerization of petrochemical products ${ }^{2}$. As aggregation processes we find such effects in medical diseases like Alzheimer's or Parkinson' $s^{3}$ and as formation processes such events are relevant to bio-engineering of novel compounds ${ }^{4}$. Yet these self-assembling processes are often influenced by a large range of external parameters like $\mathrm{pH}$, temperature, pressure, nutrients or impurities in the system. The evolving macromolecule in turn provides a wealth of parameters to investigate the growth of the molecule: spectra, time-resolved fluorescence, microscopic size and shape, magnetism or conductivity. It has proven difficult to investigate and understand these interlinked characteristics by tracking single parameters. To overcome this problem coupled multi-parameter sensing has been used with good success. Combining the excitation and the emission spectra Nighswander-Rempel and co-workers have shown a quantum yield map of synthetic eumelanin ${ }^{5}$. We now extend combined excitation and emission measurement to track chemical compounds through the formation process of eumelanin. 
In the human body eumelanin derives from the amino acid Tyrosine which is then converted by enzymatic action and oxidisation into the molecule L-Dopa to start the melanin formation pathway ${ }^{6}$. Starting the reaction directly from L-Dopa has proven to be a realistic method to investigate melanin in laboratory conditions ${ }^{7}$. Leading melanin research centres have recently made great progress in joining work to standardise nomenclature and protocols to enable comparison of procedures and results ${ }^{8,9}$.

Ground breaking work has been done on the characterisation of different forms of melanin, gaining understanding of stacking ${ }^{10}$, identifying precursor molecules ${ }^{11}$ and characterizing particle sizes ${ }^{12}$. A 'top-down' approach using readily formed melanin - be that natural or lab made - to be broken down and its components analysed often suffers from possible artifacts. Subsequently we have seen great progress in 'bottom-up' work tracking the formation process of melanin from its initial precursor molecules, binding- and stacking-steps ${ }^{13}$ and more recently identifying chemical components of the melanin macro-molecule $e^{14,15,16}$.

Melanin in all its forms of eumelanin, pheomelanin and neuro-melanin has been shown to possess a broad range of properties like ion sequestration ${ }^{17}$, paramagnetism ${ }^{18}$ and conductivity ${ }^{19}$. But melanin is first and foremost a photo-active molecule showing an intriguing broadband absorption and being an imperfect absorber - showing complex fluorescence. We therefore investigate the photoproperties of melanin to characterize its evolution from the precursor molecule L-Dopa to fully formed eumelanin. Extensive work in characterizing spectral and time resolved properties of key melanin compounds 5,6-dihydroxyindole (DHI) ${ }^{14}$ and 5,6-dihydroxyindole-2-carboxylic acid (DHICA) ${ }^{20}$ allows us to identify the contribution of these compounds to the overall melanin fluorescence signal within selected spectral regions.

\section{Material \& Methods:}

We generate eumelanin from L-Dopa as described previously ${ }^{21}$ whereby an L-Dopa stock solution is generated at $1 \mathrm{mM}$ concentration in de-ionized water, mixed in a sonication bath for 10 minutes and then stored at $21^{\circ} \mathrm{C}$ in the dark. Chemicals were obtained from Sigma Aldrich (Sigma Aldrich, UK) at the highest purity available. Measurements were done at an L-Dopa concentration of $100 \mu \mathrm{M}$. The $\mathrm{pH}$ was adjusted using ammonia leading to a reaction time from pure L-Dopa to melanin showing full spectral absorbance and emission characteristics within few hours. The Excitation \& Emission Matrices were recorded on a FluoroLog3-22 (Horiba Scientific, Edison, USA). The fluorescence lifetime decays were recorded on a DeltaFlex (Horiba Jobin Yvon IBH, Glasgow, UK).

Results:

While displaying a range of different physiological roles - some still largely unknown - melanin is first and foremost a photo-protective molecule: an absorber. The formation of melanin can be tracked by recording the evolution of its characteristic absorption spectrum.

Absorbance spectra of melanin were measured in a spectrophotometer. Experimentally the double compartment spectrophotometer consists of one or multiple steady state light sources; typically, a 
$25 \mathrm{~W}$ deuterium lamp for UV excitation $190-330 \mathrm{~nm}$ and a $20 \mathrm{~W}$ halogen lamp for Visible/NIR excitation 330-1100 $\mathrm{nm}$. The wavelength of light is selected by use of an excitation monochromator which may also be coupled to a filter wheel to remove any second order effects. When the spectrophotometer is first powered on, it should be left on for 15 minutes to allow the lamps to reach thermal equilibrium. Before any measurement the shutter of the excitation monochromator is closed and the dark counts are recorded at both photodiodes for ten times the measurement integration time. This is used to compute a dark offset which is subtracted from any subsequent measurements. Before measurement of a sample a blank scan is performed to account for any bias between the sample compartment and the baseline compartment which may be present due to a slight misalignment of the optics. If cuvettes are a non-standard geometry e.g. microcuvettes these should be placed in the sample compartment when running the blank scan to compensate for their smaller optical window. For the measurement two matching quartz cuvettes are used; the first quartz cuvette, the sample cuvette contains analyte and solvent while the second quartz cuvette, the baseline cuvette contains solvent only. The intensity of the light passing through the sample is recorded simultaneously to a baseline measurement and with the above corrections used to calculate the absorbance spectrum. It should be noted that absorbance is a ratio and hence any energy differences in energy output per wavelength of the light source or wavelength differences in the quantum efficiencies of the detectors are effectively divided out (Figure 1).

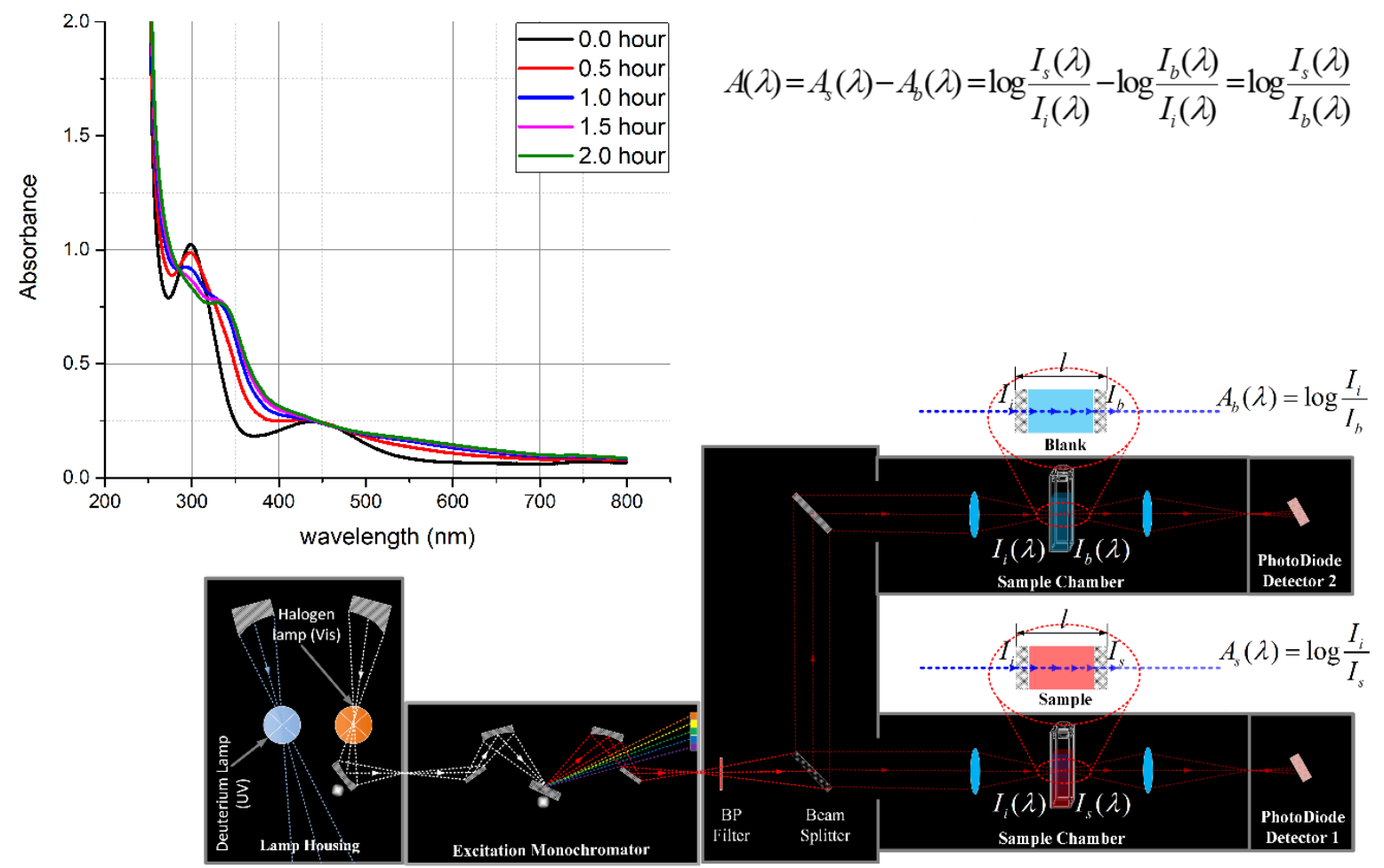

Figure 1: Experimental procedure for recording melanin absorbance

The absorbance spectra of de novo forming eumelanin initially show distinct peaks but smooth out over time. The formula illustrates how absorbance is measured from the ratio of intensities. Most standard type absorbance instruments work in line with the illustration given here. 
A finely balanced system: making eumelanin in the lab

Taking absorbance scans over time reveals several isosbestic points in the spectrum. While an isosbestic point is not in itself an indication of the conversion of one species into another or of an equilibrium between two species it does show that the stoichiometry (the relative quantity of reactants and products) remains constant during the reaction and that no secondary reactions occur during the observed time range ${ }^{22}$ With the melanin absorption spectra as it evolves during the formation process showing several isosbestic points we have clear indication of a highly interlinked formation network. We adjust the $\mathrm{pH}$ to tune the speed of the formation process. A small alteration in $\mathrm{pH}$ is sufficient to blur one isosbestic point indicating a change in the overall chemical composition (Figure 2).

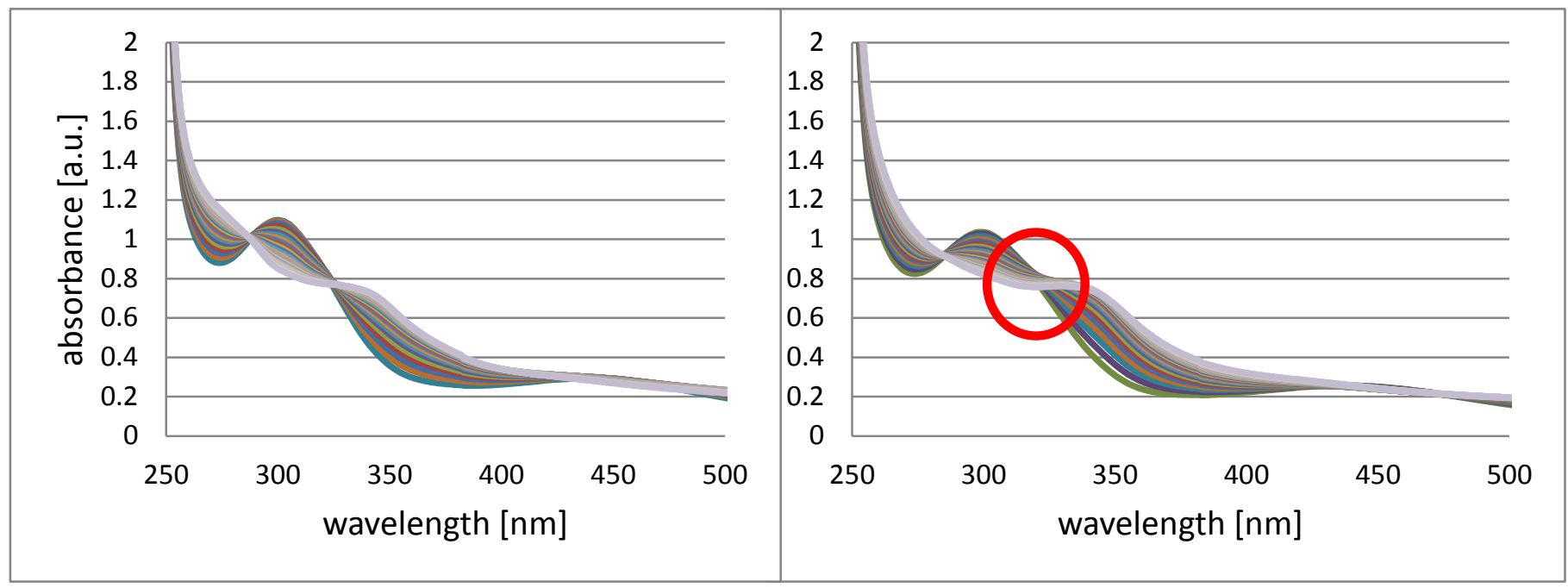

Figure 2: Evolution of the absorption spectrum of eumelanin

Shown are the absorption spectra of de novo forming melanin recorded in 5 minute intervals at $\mathrm{pH} 8$ (left) and pH 8.3 (right). The relatively small pH change leads to the blurring of the isosbestic point at $320 \mathrm{~nm}$ emission indicating a shift in the finely balanced formation process.

Coupling Parameters: Excitation-Emission Matrices (EEM)

Measuring melanin parameters separately often leaves difficulties in comparing and linking findings from different experiments. A photo-active molecule like melanin can be characterized through a wide range of parameters: its absorbance (key to its purpose as our 'natural sunscreen'), its fluorescence; (small but not at all negligible), its excitation, the size of its particles, its conductivity and many more. To gain control over this plethora of parameters we conducted coupled measurements recording melanin characteristics simultaneously.

An Excitation Emission Matrix (EEM) provides a highly detailed 'spectral fingerprint' of the melanin When an EEM is measured we observe two wavelength dimensions which can be used to separate out the individual components of the linear absorbance spectrum. The schematic (Figure 3 ) shows a 
Horiba Scientific FluoroLog 3-11 with single grating excitation and emission monochromators. The excitation optics are similar to the spectrophotometer discussed above however the light source in this case a $450 \mathrm{~W}$ xenon lamp is substantially more powerful. Approximately $8 \%$ of the light is redirected onto a reference photodiode and the remaining $92 \%$ of the light is used to excite the sample. The fluorescence emission from the sample occurs at all directions whilst excitation light is more likely to pass directly straight through the sample. In order to maximise the ratio of fluorescence measured to that of excitation light a fluorimeter is hence typically setup to collect emission at right angles. The emission monochromator then selects the emission wavelength of light reaching the photomultiplier tube S1. As in the case of the spectrophotometer an assortment of optical filters may be coupled with each monochromator in order to reduce second order effects however these are not present as standard on all fluorimeters. For both the reference photodiode $\mathrm{R} 1$ and the emission photomultiplier S1 a dark offset is measured to compensate for dark counts. Unlike absorbance the fluorescence emission is not a ratio and any spectrum measured is hence subject to the intensity of the light source for this reason the value S1/R1 is normally computed opposed to S1 on its own. Moreover the fluorimeter has a non-uniform wavelength response subject to the wavelength dependence primarily of the detector's quantum efficiency and secondary to the optics. Instrumental correction factors for emission MCorrect and excitation XCorrect are measured during the time of instrumental manufacturer by comparing the measured spectrum to the actual spectrum of standard reference materials. The instrumental correction factors allow for S1c/R1c the corrected spectrum to be measured. Emission spectra are typically obtained by parking the excitation monochromator at a specified wavelength and scanning the emission monochromator at higher wavelengths. Excitation spectra are obtained by parking the emission monochromator at a specified wavelength and scanning the excitation monochromator at lower wavelengths. The EEM on the other hand is obtained by scanning both monochromators (Figure 3). Melanin is a complicated sample and the emission at various excitation-emission pairs may differ by many orders of magnitude, sometimes plotting the EEM in a log scale is insightful so smaller components are emphasised. Although the EEM contains a vast amount more information than individual line spectrum one major drawback of the EEM is the acquisition time required especially in the case of melanin formation which has a relatively rapid reaction. This may be compensated by use of a larger wavelength increment and smaller integration time. The smaller integration time will however lower the signal to noise ratio which becomes problematic when wavelength correction factors are used especially near the limits of the detector's working range. The signal to noise ratio can partially be addressed by increasing the monochromator bandpass although this comes at a sacrifice of spectral resolution. In this work a FluoroLog 3-22 with double grating excitation and emission monochromators was used. The bandpasses of each monochromator were set to $5 \mathrm{~nm}$ and a series of emission scans were measured from $300-800 \mathrm{~nm}$ in $5 \mathrm{~nm}$ steps. The initial excitation wavelength was $250 \mathrm{~nm}$ and stepped up in $5 \mathrm{~nm}$ steps after each emission scan until the upper excitation wavelength of $700 \mathrm{~nm}$. An integration time of $0.4 \mathrm{~s}$ was used resulting in an overall acquisition time of $\sim 40$ minutes for the EEM. This is substantially larger than differences recorded from line spectra however the EEM remains sufficient to highlight excitation-emission pairs of species present in melanin (Figure 3). 

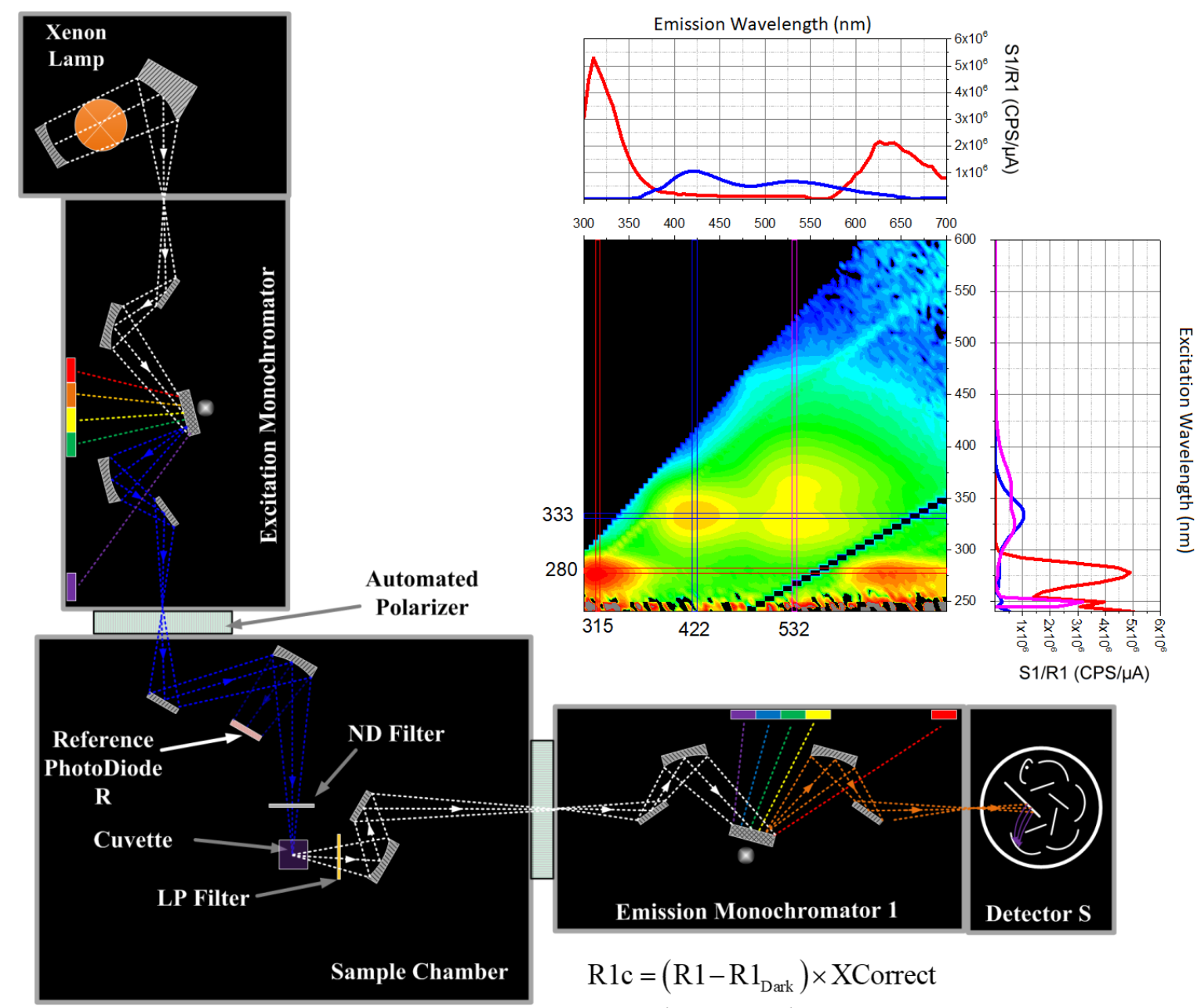

$$
\begin{aligned}
& \mathrm{R} 1 \mathrm{c}=\left(\mathrm{R} 1-\mathrm{R} 1_{\text {Dark }}\right) \times \mathrm{XCorrect} \\
& \mathrm{S} 1 \mathrm{c}=\left(\mathrm{S} 1-\mathrm{S} 1_{\text {Dark }}\right) \times \mathrm{MCorrect}
\end{aligned}
$$

Figure 3: Configuration for the recording of an EEM

Recording an EEM of melanin forming de novo from L-Dopa takes about 40 minutes in the configuration we used. The EEM shown is recorded about midway through the melanin formation with some of the L-Dopa still prominent at a spectral area around $280 \mathrm{~nm}$ excitation and $315 \mathrm{~nm}$ emission but we already observe further fluorescent structures. The EEM is plotted out in log scale in order to place an emphasis on smaller components. A contour profile plot may be used to show linear emission scans at the excitation wavelengths of interest $280 \mathrm{~nm}$ and $333 \mathrm{~nm}$ (top: blue \& red) in addition to linear excitation scans at emission wavelengths of interest 315, 412 and $532 \mathrm{~nm}$ (right: blue, red \& pink). The illustration shows the instrument setup for an EEM.

Areas of Interest: Identifying building sites of melanin

Investigating an EEM recorded during the formation process of melanin allows identifying specific sites where the absorption generates significant fluorescence emission (Figure 3). Some of these areas show a transient character suggesting that fluorescent structures are later transformed or integrated into non-fluorescent parts of the molecule other developing areas of interest stabilize over the formation process. 
The spectral area excited around $280 \mathrm{~nm}$ and emitting at $315 \mathrm{~nm}$ is most likely related to L-Dopa absorption and emission. The slightly broader emission around this region suggests that we observe small variations in chemical composition of the molecules contributing to this region.

Excitation at $333 \mathrm{~nm}$ reveals two areas of emission; one around $422 \mathrm{~nm}$ and a second weaker emitting region around $532 \mathrm{~nm}$. It is an interesting feature that both emission peaks are excited at the same wavelength. The chemical structure of the evolving melanin might retain for some time a favoured absorption line with incomplete energy dissipation capacities leaving two fluorescent spectral 'leaks'.

Tracking pieces of a puzzle: characteristic lifetimes in the EEM

Excellent work has been done to characterize single building blocks of melanin ${ }^{14,20}$. We can use these characterisations to identify components of melanin present during the formation process. Timeresolved fluorescence data give an insight into such complex fluorescence. From the EEM (Figure 3) one area of interest can be identified in the excitation-emission pair of $333 \mathrm{~nm}$ and $422 \mathrm{~nm}$ respectively. We used time correlated single photon counting (TCSPC) to investigate the fluorescence characteristics of newly forming melanin at the identified spectral area of interest. Fluorescence decays are characteristic for a given fluorophore. The fluorescence decay time is highly dependent on the immediate surrounding of the fluorophore. Fluorescence lifetime decay recording has been used as a 'molecular ruler' measuring molecular characteristics on the nm scale giving insight into small changes in the chemical structure of a fluorophore. Measuring fluorescence decay times in identified areas of interest during the formation process yields an additional dynamic component to the measurement.

This work uses a DeltaFlex; for excitation a DeltaDiode $340 \mathrm{~nm}$ pulsed LED is selected, the TDM-800 monochromator is set to $422 \mathrm{~nm}$ with a bandpass of $8 \mathrm{~nm}$ and a PPD 850 photomultiplier tube detector is selected. In order to achieve maximum throughput DeltaHub timing electronics are used with a time range of $200 \mathrm{~ns}$. Every $30 \mathrm{~s}$ a multi-exponential fluorescence decay is recorded. First observations by eye show that these decays have substantially different intensity and decay characteristics. Initially a short lived component is seen to be prevalent and its intensity increases with respect to time. Subsequently the prevalence of this component begins to fall and the formation of longer lived components is observed. Time resolved fluorescence data allows for an analysis of intensity of the individual components to give a better understanding of melanin's complex self-assembly (Figure 4). 

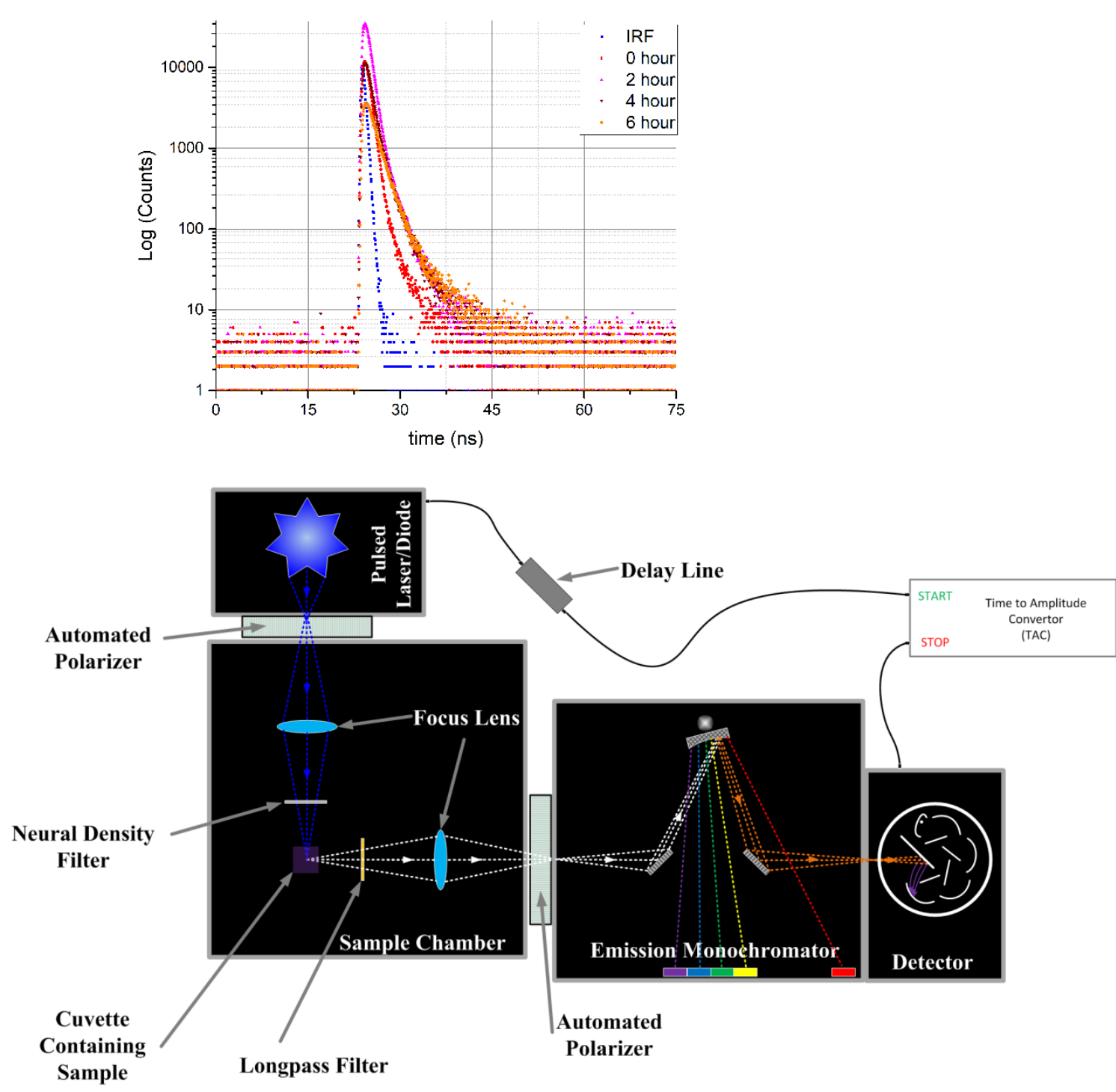

Figure 4: Equipment for recording the fluorescence decay times in a given spectral area of interest

Fluorescence lifetime decays taken at given times during the melanin formation in an area of interest identified in the EEM at $333 \mathrm{~nm}$ excitation and $422 \mathrm{~nm}$ emission. The illustration shows a typical TCSPC setup.

For small decay times the data obtained via a fluorescence lifetime measurement is convoluted with the instrumental response function $P(t)$ meaning one cannot directly obtain the desired decay parameters directly from the data.

$$
P(t) \otimes T(t)=F(t)
$$

Rayleigh scattering occurs on the fs time-scale which is instantaneous with respect to the typical psns instrument response function $P(t)$ of a time resolved fluorimeter and as a consequence any attempt to measure Rayleigh scattering will yield the instrumental response function $P(t)$. To optimise Rayleigh scattering, a dilute solution of colloidal silica LUDOX is used in place of the sample 
and as Rayleigh scattering is elastic the emission monochromator is set to the same wavelength of the excitation source. To compensate for any wavelength dependence of the transit spread time of the photomultiplier.due to the decay $F(t)$ being measured at a higher wavelength an additional shift parameter is used. This shift parameter is typically very small and not quoted in literature. Neutral density filters are placed in the sample compartment to ensure that the hit rate (Stop/Start) $<2 \%$ to avoid photon pile-up. Re-convolution analysis is then applied. Re-convolution analysis relies on the correct theoretical model $T(t)$ to be selected to fit the data. Typically, $T(t)$ is series of exponentials with associated decay times and pre-exponential factors. The first "guess" is usually the simplest case; a mono-exponential then the complexity is gradually built up until a satisfactory fit is achieved (Equation 2). The HORIBA Scientific EzTime acquisition and analysis software allows up to 5 exponentials to be fitted.

$$
T_{x}(t)=b_{1} \exp \left(-\frac{t}{\tau_{1}}\right)+\ldots+b_{x} \exp \left(-\frac{t}{\tau_{x}}\right)
$$

The goodness of fit criterion $\chi^{2}$ and Poissonian statistics are then used to compare $F(t)$ to $P(t) \otimes T(t):$

$$
\chi^{2}=\sum_{\text {Data pts }}\left\{\frac{F(t)-P(t) \otimes T(t)}{[F(t)]^{1 / 2}}\right\}^{2}
$$

For a good model the residuals have to be random and the $\chi^{2}$ value has to be close to 1.0 preferably $0.9 \leq \chi^{2} \leq 1.25$. For non-random residuals the bias of the residuals allows one to see if they need to fit an extra exponential component or not and whether this component is positive or negative. When fitting there are several parameters which are useful $\tau_{1}, \ldots, \tau_{i}$ are the decay times of the sample, typically quoted in ns with a standard error of 3 standard deviations i.e. has a confidence interval of $\sim 99 \%$. The raw pre-exponential factors $b_{1}, \ldots, b_{\text {j }}$ show the relative contribute of each decay component towards the peak of the decay. In this work global analysis is utilised; in the first step all the fluorescence decays are summed and fitted to obtain global decay times. These global decay times are then applied to each individual decays to give a readout of $b_{1}, \ldots, b_{\mathrm{j}}$ with respect to reaction time. For chemical reactions such as the formation of melanin these pre-exponentials will change with respect to reaction time and can be used to highlight the reaction and formation of starting species, the presence of an intermediate and the formation of a final product.

Each fluorescence decay recorded during the melanin formation is generated from a composition of decay times characteristic for given fluorescent compounds present at the moment of recording. When we track the relative contribution of these components we observe a complex pattern with both the initial rise of the fluorescence intensity and its subsequent decline as present in the rise and fall of the photon peak count rate over time (Figure 4). More so the data show the emergence of more complex structures contributing to the fluorescence. The fluorescence decay function can be described by a function comprising three exponentials with time constants of $0.61+/-0.01 \mathrm{~ns}, 1.79$ +/- 0.07ns and a longer component of 6.6 +/-0.8ns (Figure 5). 

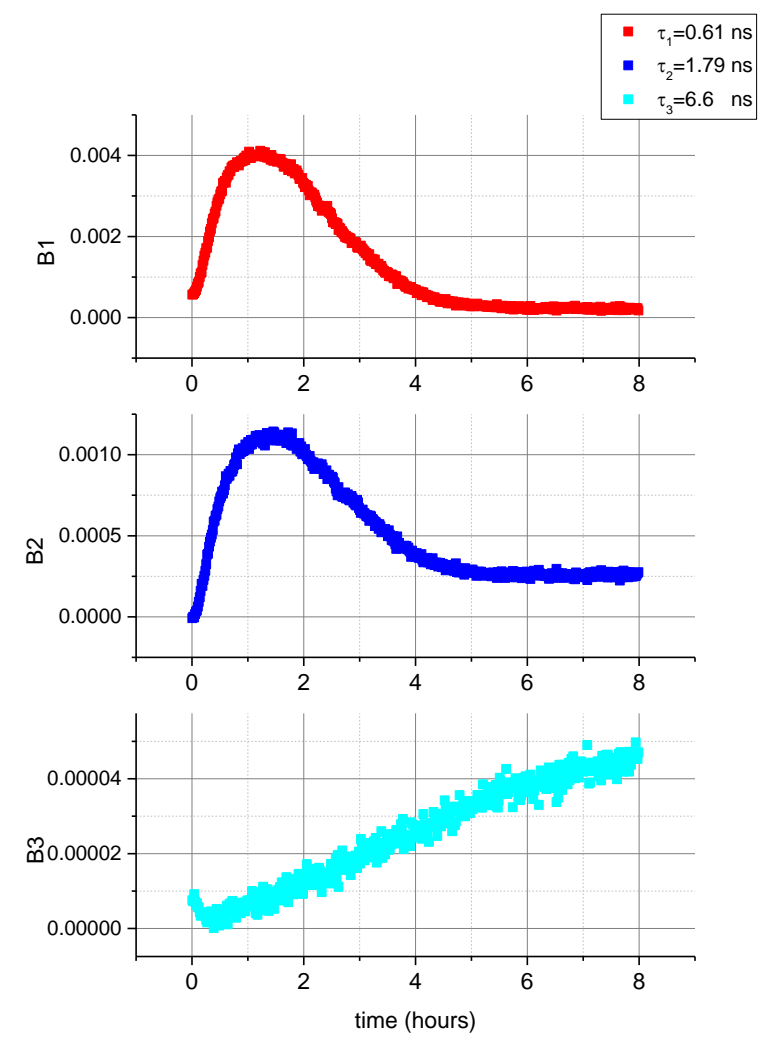

Figure 5: Evolution of lifetimes

Tracking the evolution of fluorescent lifetimes in areas of interest allows identifying the presence of characteristic melanin components. Following the relative contribution of the identified component over the duration of the formation process reveals which fluorescent components become integral parts of melanin and which ones are transient elements of the formation process.

Interestingly the long lifetime component trackable very early in the formation process can also be measured in fully formed melanin ${ }^{23}$. The contribution of this decay component rises steadily over the melanin formation process. The contribution of the two short components rises with the melanin formation and then declines again. One of these fluorescent molecules with a decay time of $0.61+/-0.01$ ns shows a strong contribution to the overall signal during the first 2 hours of the melanin formation but then its contribution completely vanishes. This could indicate a chemical modification of the molecule during the formation or the evolution of a non-radiative decay path for the energy absorbed by this structure. The second component exhibiting transient fluorescence displays a decay time of $1.79+/-0.04 \mathrm{~ns}$. With the fluorescence lifetime being highly susceptive to small modification in the fluorophore or the direct environment around it, the fluorescence decay time provides a good spectral identifier for a given molecule and this fluorescence lifetime and the emission wavelength match very well with parameters identified by Corani and co-workers characterizing the melanin building block $\mathrm{DH}^{14}$. This is a strong suggestion that we are looking at a contribution from $\mathrm{DHI}$ at this given excitation-emission area.

Both the short transient and the longer decay times in the region investigated do not offer direct attributions to identified melanin building blocks. The key element DHICA has also been well 
characterized in spectral and time resolution ${ }^{20}$. Its characteristic lifetimes do not provide a match for this excitation-emission area.

Closer to nature: effecting melanin's structure

The production of eumelanin in the lab is a well-established technique yielding highly reproducible results. Yet melanin extracted from natural sources does show small but intriguing differences to lab generated melanin. The addition of micro-nutrients like transition metal ions to the reaction can alter the characteristics of the melanin generated ${ }^{24,25}$. Using EEMs we identify differences between lab generated and naturally occurring melanin and show an example how the addition of a micro nutrients can lead to a melanin closer in characteristics to the natural form (Figure 6).

a)

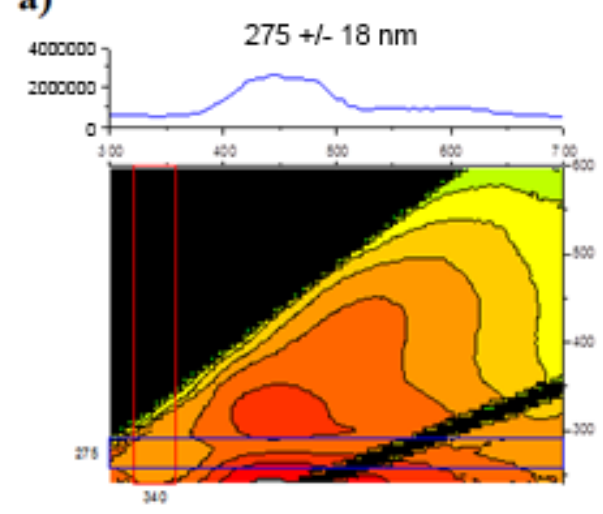

c)

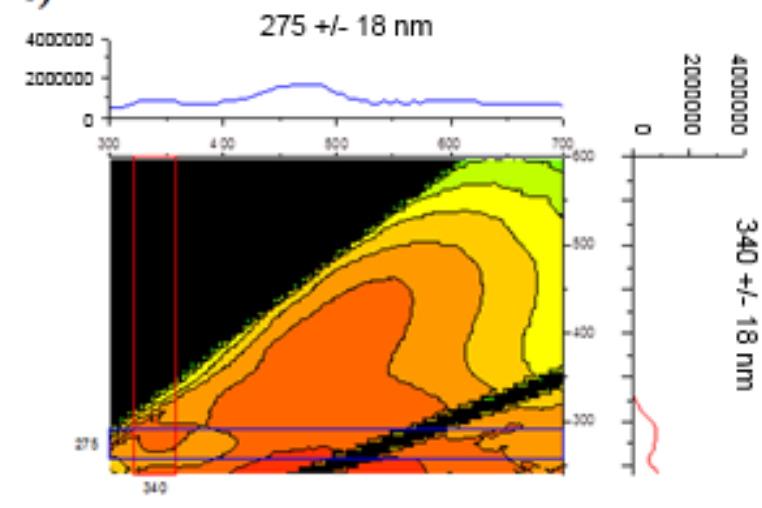

b)

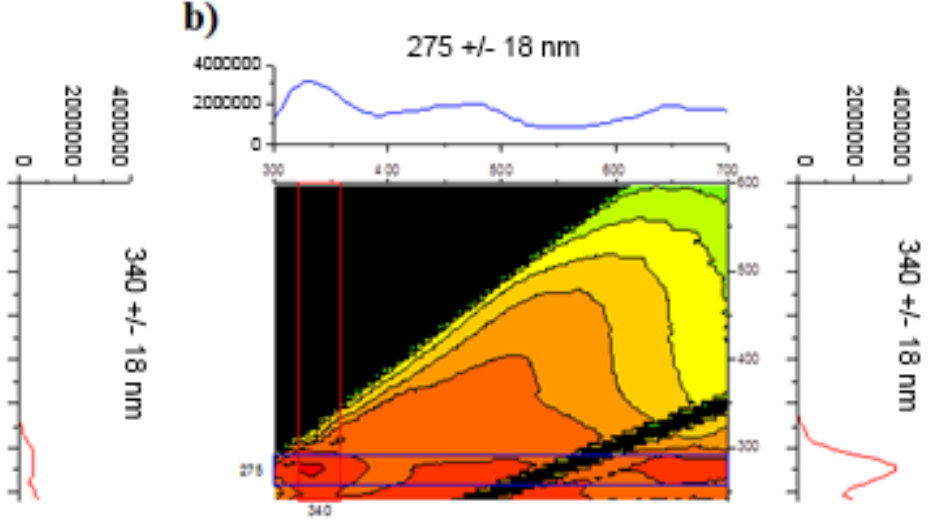

Figure 6: The effect of micro-nutrients on EEMs

Comparing the EEM of laboratory melanin (a) with that of natural sepia melanin (b) we find characteristic differences like a short wavelength area present in natural melanin but not in lab melanin (ex: $275 \mathrm{~nm}$, em:340nm). Addition of $10 \mu \mathrm{M}$ copper ions partially restores this area (c).Adjacent graphs show the excitation (blue) and emission (red) framing the area of interest.

The EEMS do striking similarities in the excitation-emission qualities of synthetic and natural melanin. In both cases the EEM is dominated by a broad emission around $470 \mathrm{~nm}$ as well as fluorescence excited in the UV region. But the EEM also reveals differences: Melanin derived from 
Sepia Officinalis exhibits a striking excitation-emission region absorbing around $275 \mathrm{~nm}$ with a relatively narrow fluorescence band around $340 \mathrm{~nm}$ emission. This feature is absent in the lab generated melanin suggesting that the lab melanin is in this feature a too simplistic analogue. The absence of this peak could be either due to missing absorbing structures formed in the lab or because of more complete energy dissipation diminishing the radiative decay path in this spectral region.

Synthetic melanin generated in the presence of $10 \mu \mathrm{M}$ copper ions generates an EEM that shows the distinctive excitation-emission at $275 \mathrm{~nm}$ and $340 \mathrm{~nm}$ partially restored. The presence of $\mathrm{Cu}^{2+}$ yields an EEM indicative of melanin with more natural spectral characteristics.

Discussion:

Melanin is involved in a multitude of physiological reactions the complexity of which we only begin to understand ${ }^{26}$. The molecule offers great opportunities as a bio-engineering tool to build bio circuitry $^{19}$, in sensing or as an immuno-neutral cover for implants or nano-medical compounds ${ }^{27}$. To fully exploit these opportunities we need greater understanding of melanin formation. Measuring coupled characteristic parameters during the formation process of complex macro-molecules opens opportunities to identify and track separate building blocks of the molecule throughout a complex reaction.

We use copper ions as an example to show how the presence of effectors during the formation process can create small variations in the melanin structure subsequently picked up in a coupledmeasurement. lons are known to interact with melanin ${ }^{17}$ and indeed become part of the melanin structure $^{28}$. Especially the interaction of iron and copper with neuro-melanin in the substantia nigra region of the brain plays an important role in the onset of neuro-degenerative disease ${ }^{29}$. Copper ions are the strongest catalyst in the conversion of dopachrome (DC) to DHICA ${ }^{30}$. Synthetic melanin generated in the presence of copper yields a simpler fluorescence decay when excited at $450 \mathrm{~nm}$ and emission is observed at $480 \mathrm{~nm}$ or $530 \mathrm{~nm}$. This indicates a possibly more ordered, more homogeneous structure ${ }^{22}$. Extensive work by Ito and co-workers has revealed how copper shifts the production of DHI and DHICA from DC strongly favouring DHICA ${ }^{25}$.

Comparing the EEM of eumelanin made from L-Dopa with one from natural Sepia melanin we find a characteristic area of interest at short excitation and emission wavelengths that is missing in the laboratory derived eumelanin. Adding copper to the formation process goes some way in recreating that excitation-emission feature present in natural melanin. This is most likely due to a shift in the balance between DHI and DHICA ${ }^{25}$. The more complex EEM from Sepia officinalis melanin used in this study, the diversity often found in studies of different naturally occurring melanin ${ }^{31}$ as well as recent work identifying how small chemical differences lead to changes in the overall appearance of melanin $^{32}$ portrait melanin as a multi-characteristic group of macro-molecules easily adapting to diverse physiological tasks and environmental conditions ${ }^{8}$. Using melanin effectively in bioengineering will require detailed understanding of the structure-function relation of melanin parts and components. Great progress has been made in identifying components ${ }^{14,20}$ and the influence of structural elements ${ }^{32}$ leading towards modification of melanin bioengineering ${ }^{33}$. Creating data 
matrices using joined-measurements of key parameters of melanin opens new opportunities to follow the generation and inclusion of key molecules into melanin.

\section{Conclusion:}

Unravelling networks of formation of aggregation processes within a complex physiological background is of immense importance. The formation of plaques and fibrils in neuro-degenerative diseases, understanding the formation of macromolecules like lignin or melanin for bioengineering or the controlled generation of polymer structures in chemical engineering require detailed insight into binding processes and intermediate compounds.

Coupling measurements generates a multi-dimensional 'parameter-landscape'. Such data array then allows the identification of key reaction sites for further investigation; reaction sites that observation of a single measurand might miss. In melanin formation from L-Dopa we present an example of a formation network. Taking measurements of the single measurand absorbance we illustrate the complexity of the formation. Coupling measurements then generates inroads for further investigation. We use a measurand array of excitation and emission for the identification of areas of interest. Tracking the temporal evolution of the fluorescence decay times in one area of interest over time allows the identification of a building block of melanin, reveals at what stages in the formation it is generated and shows its contribution to the final melanin fluorescence. Such synchronous recording of parameters opens insights into complex formation processes.

Going back to the measurand array of excitation and emission we also have a tool to compare melanin created in the lab with a natural melanin. Identifying characteristic differences we use a physiologically relevant micro-nutrient to generate a melanin closer to the natural form. Research instrumentation undergoes continuous improvement and development in turn progressing our understanding of complex physiology or chemistry. Yet to get insight into finely balanced networks of formation it proves a very promising approach to couple measurements of different characteristic parameters creating multi-dimensional arrays of measurands. This gives a worthwhile addition and indeed extension to single parameter observation.

Acknowledgement:

The authors thank the EPSRC and Innovate UK for their support. The authors have declared that no conflicting interests exist.

References:

1 Chianelli RR, Siadati M, Mehta A, Pole J, Carbognani Ortega L \& Chiang LY Self-aasembly of asphaltene aggregates: Synchrotron, simulation and chemical modelling techniques applied to problems in the structure and reactivity of asphaltenes Asphaltenes, Heavy Oils and Petroleomics Mullins OC, Sheu EY, Hammarni A \& Marschall AG Eds. Springer, New York, 375-400, 2007 

block copolymer meshes by orthogonal self-assembly Nature Communications, 7:10518, 2016

Li Y, Zhao J \& Hölscher C Therapeutic potential of baicalein in Alzeimer's disease and Parkinson's disease CNS Drugs doi: 10.1007/s40263-017-0451-y. 2017

Qiu Z, Han T, Lam JWY \& Tang BZ Recent new methodologies for acetylenic polymers with advanced functionalities Topics Current Chem. 375(4) 702017

Nighswander-Rempel SP, Riesz J, Gilmore J \& Meredith P A quantum yield map for synthetic eumelanin J Chem Phys. 123(19) 1949012005

Kamakshi R Fairness via formulations: a review of cosmetic skin-lightening ingredients J Cosmetic Science 63 43-54 2012

Ito S A chemist's view of melanogenesis. Pigment Cell Research 16 (3), 230, 2003

d'Ischia M, Wakamatsu K, Napolitano A, Briganti S, Garcia-Borron J-C, Kovacs D, Meredith P, Pezzella A, Picardo M, Sarna T, Simon JD \& Ito S Melanins and Melanogeneisis: Methods, Standards, Protocols Pigmen Cell \& Melanoma Research 26(5) 616-633 2013

9 d'Ischia M, Wakamatsu K, Cicoira F, Di Mauro E, Garcia-Borron J-C, Commo S, Glavan I, Ghanem G, Kenzo K, Meredith P, Pezzella A, Santato C, Sarna T, Simon JD, Zecca L, Zucca FA, Napolotano A \& Ito S Melanins and Melanogenisis: from Pigment to Human Health and Technological Application Pigment Cell \& Melanoma Research 28(5) 520-544 2015

10 Zajac GW, Gallas JM, Cheng J, Eisner M, Moss SC \& Alvarado-Swaisgood AE The fundamental unit of synthetic melanin: a verification by tunnelling microscopy of $X$-ray scattering results Biochim Biophys Acta 1199 271-278 1994

11 Meng S \& Kaxiras E Theoretical models of eumelanin protomolecules and their optical properties Biophys J. 94(6) 2095-2105 2008

12 Büngeler A, Hämisch B, Huber K, Bremser W \& Strube OI Insight into the final step of the supramolecular buildup of eumelanin Langmuir doi 10.1021/acs.langmuir.7b01634. 2017

13 Stark KB, Gallas JM, Zajac GW, Golab JT, Gidanian S, McIntire T \& Farmer PJ Effect of stacking and redox state on optical absorption spectra of melanins - comparison of theoretical and experimental results J Phys Chem B 109 1970-1977 2005

14 Corani A, Huijser A, ladonisi A, Pezzella A, Sundström V \& d'Ischia M. Bottom-Up Approach to Eumelanin Photoprotection: Emission Dynamics in Parallel Sets of Water-Soluble 5,6Dihydroxy-Indole Based Model Systems. Journal of Physical Chemistry B 116(44), 1315113158, 2012

15 Chen C-T, Ball V, Gracio JJA, Singh MK, Toniazzo V, Ruch D \& Buehler MJ Self-assembly of tetramers of the 5,6-dihydroxyindole explains the primary physical properties of eumelanin: experiment, simulation and design ACS Nano 7(2) 1524-1532 2013 
16 Prampolini G, Cacelli I \& Ferretti A Intermolecular interactions in eumelanins: $a$ computational bottom-up approach. I. small building blocks RSC Advances 5385132015

17 Larson B \& Tjalve H Studies on the melanin-affinity of metal ions Acta Physiol Scand 104 4794841978

18 Chio SS, Hyde JS \& Sealy RC Paramagnetism in Melanins: $p H$ Dependence Archives Biochemistry Biophysics 215(1), 100-106, 1982

19 Mostert AB, Powell BJ, Pratt FL, Hanson GR, Sarna T, Gentle IR \& Meredith P Role of semiconductivity and ion transport in the electrical conduction of melanin PNAS 109(23) 8943-8947 2011

20 Corani A, Pezzella A, Pascher T, Gustavson T, Markovitsi D, Huijser A, d'Ischia M \& Sundström V. Excited-State Proton-Transfer Processes of DHICA Resolved: From Sub-Picoseconds to Nanoseconds Journal of Physical Chemistry Letters 4 1383-1388, 2013

21 Sutter JU \& Birch DJS Metal Ion Influence on Eumelanin Fluorescence and Structure Methods and Applications in Fluorescence 20240052014

22 Greger M, Kollar M \& Vollhardt D Isosbestic points: how a narrow crossing region of curves determines their leading parameter dependence Phys Rev B. 87(19) 1951402013

23 Berezin MY \& Achilefu S Fluorescence Lifetime Measurements and Biological Imaging Chemical Review 110(5), 2641-2684, 2010

24 Birch DJS \& Sutter JU The Effect of Copper on Eumelanin Photophysics and Morphology, Proceeding of SPIE 85878587052013

25 Ito S, Suzuki N, Takebayashi S, Commo S \& Wakamatsu K Neutral pH and Copper lons promote Eumelanogenesis after the Dopachrome Stage Pigment Cell \& Melanoma Research 26(6) 817-825 2013

26 Haining RL \& Achat-Mendes C Neuromelanin, one of the most overlooked molecules in modern medicine, is not a spectator Neural Regen Res. 12(3) 372-375 2017

27 Hong SH, Sun Y, Tang C, Cheng K, Zhang R, Fan Q, Xu L, Huang D, Zhao A \& Cheng Z Chelatorfree and biocompatible melanin nanoplatform with facile-loading gadolinium and copper-64 for bioimaging Bioconjug Chem doi: 10.1021/acs.bioconjchem.7b00245. 2017

28 Ball V, Bour J \& Michel M Step-by-step deposition of synthetic dopamine-eumelanin and metal ions J Colloid Interface Sci. 405 331-335 2013

29 Double KL Neuronal vulnerability in Parkinson's disease Parkinsonism Relat Discord Suppl 1 S52-S54 2012

30 Palumbo A, d'Ischia M, Misuraca G \& Prota G Effect of Metal lons on the Rearrangement of Dopachrome Biochim Biophys Acta 925203-209 1987 
31 Alaluf S, Atkins D, Barrett K, Blount M, Carter N \& Heath A Ethnic variation in melanin content and composition in photoexposed and photoprotected human skin Pigment Cell Res. 15(2) 112-118 2002

32 Micillo R, Panzella L, Koike K, Monfrecola G, Napolitano A \& d'Ischia M "Fifty Shades" of black and red or how carboxyl groups fine tune eumelanin and pheomelanin properties Intern J Molecular Sci. 17(5) E746 2016

33 d'Ischia M \& Messersmith PB From sequence to colour Science 356(6342) 1011-1012 2017 